\title{
Effect of long period treatment with erythropoiesis stimulating agents on clinically and laboratory parameters in hemodialysis autosomal dominant polycystic kidney disease patients
}

\author{
Olga Hilda Orăsan ${ }^{1 * \#}$, Ljubomir Petrov ${ }^{2}$, Laura Urian ${ }^{2 \#}$, Angela Cozma ${ }^{1}$, \\ George Ciulei ${ }^{1}$, Ioan Mihai Patiu ${ }^{3}$, Remus Aurel Orăsan ${ }^{3}$ \\ 1. $4^{\text {th }}$ Medical Department, University of Medicine and Pharmacy Iuliu Hatieganu, \\ Cluj-Napoca, Romania \\ 2. Hemathology Department, University of Medicine and Pharmacy Iuliu Hatieganu, \\ Cluj-Napoca, Romania \\ 3. Nefromed Dialysis Center, Cluj-Napoca, Romania
}

\begin{abstract}
Introduction. The study of dialysis patients not needing erythropoiesis-stimulating agents (ESA) for long periods of time has gained interest lately. The aim of this study was to compare laboratory and clinical parameters in hemodialysis patients with autosomal dominant polycystic kidney disease (ADPKD) treated or not with ESA. Methods. Forty-six hemodialysis ADPKD patients were studied for 8 months and they were divided into: group 129 patients who received ESA during the study period and group 2- 17 patients with no ESA treatment. The following parameters were determined: weekly treatment time, body mass index (BMI), pre-session diastolic blood pressure $(D B P)$, pre-session systolic blood pressure (SBP), blood volume processed (BVD), interdialytic body weight gain (IBWG), spKt/V-K/DOQI formula $(K t / V)$, urea distribution volume (UDV), hemoglobin (Hb), ferritin, transferrin saturation (TSAT), serum phosphate, total serum calcium, normalized protein catabolic ratio (nPCR), albumin, and intact parathormone (PTH). Results. Patients not requiring ESA were more likely to be men, had higher Hb, albumin, total serum calcium levels, IBWG, UDV, BVP, and weekly treatment time. They had lower ferritin, TSAT, $S B P$. There was no difference regarding DBP, BMI, serum phosphate, PTH, Kt/V, and $n P C R$. Conclusion. Hemodialysis ADPKD patients not treated with ESA seem to be better nourished, with a slightly better SBP control, with longer dialysis time and increased $\mathrm{Hb}$ (despite lower iron loading markers), compared to hemodialysis ADPKD patients treated with ESA.
\end{abstract}

Keywords: autosomal dominant polycystic kidney disease, erythropoiesis-stimulating agents, anemia, hemodialysis

Received: 23 $3^{\text {rd }}$ March 2017; Accepted: $4^{\text {th }}$ September 2017; Published: $6^{\text {th }}$ October 2017

\footnotetext{
* Corresponding author: Olga Hilda Orăsan, University of Medicine and Pharmacy Iuliu Hatieganu ClujNapoca, Romania. E-mail: olgaorasan@gmail.com

\# Olga Hilda Orăsan and Laura Urian equally contributed to this paper.
} 


\section{Introduction}

A relatively small part of the chronic hemodialysis (HD) patients maintain acceptable levels of hemoglobin $(\mathrm{Hb})$ without requiring erythropoiesis-stimulating agents (ESA) or blood transfusions over longer periods of time (1). Naturally occurring $\mathrm{Hb}$ concentrations above 12 $\mathrm{g} / \mathrm{dL}$ do not associate with increased mortality in HD patients. The absence of anemia may be related, in part, to a higher capacity of patients to produce erythropoietin (EPO) (2). The presence of renal or hepatic cysts was associated with increased EPO synthesis and the absence of anemia in patients on HD (3). Patients with polycystic kidney disease produce more EPO and require lower ESA doses (4). Cyst stromal cells produce mRNA for EPO, and the cyst EPO production is independent of oxygen pressure (5). In HD patients with autosomal dominant polycystic kidney disease (ADPKD) the clinical and laboratory factors associated with the presence or the absence of the ESA therapy are less investigated.

The objective of this study was to compare clinical and laboratory features of HD patients with ADPKD not requiring ESA for a long period of time (8 months) with ADPKD patients on HD who received ESA during the same period of time.

\section{Subjects and Methods}

We performed a multicenter, retrospective and observational study, which included patients from 7 Romanian dialysis centers.

All patients with ADPKD and end-stage renal disease (ESRD) on regular HD treatment were included in the study. Patients with acute clinical conditions requiring hospitalization, patients with missing laboratory or clinical data during the study period were excluded from the study. A total of 46 patients $(8.9 \%$ of the total number of patients) met the inclusion and the exclusion criteria. Only patients who gave their informed consent were included in the study.

The patients were treated with bicarbonate buffered high-flux HD using polysulphone, synthetic, high flux dialyzers (Fresenius Medical Care, Bad Homburg, Germany). Water quality was regularly monitored to ensure tight bacteriologic standards (total viable count $<0.1 \mathrm{cfl} / \mathrm{mL}$ and endotoxin level $<0.03 \mathrm{EU} / \mathrm{mL}$ ).

For all patients in the study group we assessed weekly treatment time, body mass index (BMI), pre-session diastolic blood pressure (DBP), pre-session systolic blood pressure (SBP), blood volume processed (BVD), interdialytic body weight gain (IBWG), spKt/V -K/ DOQI formula $(\mathrm{Kt} / \mathrm{V})$, urea distribution volume (UDV), hemoglobin ( $\mathrm{Hb})$, serum phosphate, total serum calcium and normalized protein catabolic ratio (nPCR), on monthly basis (first midweek dialysis of each month). Every 3 months (first midweek dialysis of the month) ferritin, transferrin saturation (TSAT), and albumin were determined, while intact parathormone (PTH) was assessed at 6 month intervals.

The 46 patients were divided into 2 study groups: the patients who received ESA during any moment in the 8 months of the study were included in group 1, and the patients who did not receive ESA at all during the entire study period formed group 2.

We compared a pooled mean of patient parameters in group 1 with a pooled mean of patient parameters in group 2.

The anemia treatment with ESA and iron and the treatment for mineral bone disease secondary to chronic kidney disease with phosphate binders (calcium-containing and calcium-free) and vitamin D derivatives were used. All centers had common medical guidelines and treatment protocols for dialysis adequacy, anemia, mineral bone condition, nutrition, and high blood pressure. ESA average monthly dose in group 1 was 4347.6 IU per patient. In group 1 intravenous 
iron was administered in 18 patients at least for one month and the average dose was $366.3 \mathrm{mg} /$ month/patient, while in group 2 only 4 patients needed iron and the average dose was $290.9 \mathrm{mg} /$ month/patient.

The study protocol was approved by the Ethics Committee of the involved dialysis centers.

Normally distributed variables were expressed as mean \pm standard deviation, while non-normally distributed variables (ferritin, $\mathrm{PTH}$, dialysis vintage, and weekly treatment time) were described as median (Q1-Q3). A p-value $<0.05$ was considered to be statistically significant. Student's t-test for independent normally distributed variables, Wilcoxon test for non-normally distributed parameters and Pearson's Chi-squared test for factors were applied. A multiple regression model was used for identifying predictive factors for ESA requirement. All statistical calculations were made using the statistical software Rcmdr Version 3.3.2.

Ethical approval: All procedures performed in the study involving human participants were in accordance with the ethical standards of the institutional and national research committees and with the 1964 Helsinki Declaration and its later amendments or comparable ethical standards.

Informed consent: The subjects' participation in the study was voluntary, biological samples were collected after obtaining the written consent for participation.

\section{Results}

In group 1, there were 10 men (34.48\%) and 19 women $(65.52 \%)$. The mean age was $58.67 \pm 9.95$ years (women $57.66 \pm 9.34$ years; men $60.59 \pm 11.27$ years) and the mean dialysis vintage was 41.06 (31.73-71.85) months (women 33.51 (23.49-71.85) months; men 47.96 (37.01-76.94) months).

Group 2 included 13 men (76.47\%) and 4 women $(23.53 \%)$. The mean age was $59.80 \pm 6.35$ years (women $59.42 \pm 5.49$ years; men $59.92 \pm 6.80$ years) and the mean dialysis vintage was 47.08 (32.49-84.53) months (women 48 (34.46-82.46) months; men 47.08 (30.94-84.53) months).

There was no statistically significant difference between the two groups in terms of age $(p=0.14)$ and dialysis vintage $(p=0.06)$, but there was a significant difference in sex distribution $(p=0.006)$.

SBP, ferritin, CRP, and TSAT were significantly higher in group 1 compared to group 2, while IBWG, albumin, $\mathrm{Hb}, \mathrm{Ca}, \mathrm{UDV}$, and BVP were lower (Table 1).

Male patients included in group 2 had statistically significant higher levels of albumin (group $1=3.6 \pm 0.46 \mathrm{~g} / \mathrm{dL}$ vs group $2=3.8 \pm 0.27$ $\mathrm{g} / \mathrm{dL}, \mathrm{p}=0.03$ ), BMI (group $1=25.1 \pm 4.34 \mathrm{~kg}$ / $\mathrm{m}^{2}$ vs group $\left.2=27.4 \pm 3.09 \mathrm{~kg} / \mathrm{m}^{2}, \mathrm{p}<0.001\right), \mathrm{Ca}$ (group $1=8.4 \pm 0.88 \mathrm{mg} / \mathrm{dL}$ vs group $2=8.9 \pm 0.85$ $\mathrm{mg} / \mathrm{dL}$, $\mathrm{p}=0.002$ ), and $\mathrm{Kt} / \mathrm{V}$ (group $1=11.2 \pm 1.6$ vs group $2=13.1 \pm 1.48, \mathrm{p}<0.001)$, and significantly lower ferritin (group $1=345.5$ (104.5828.7) $\mathrm{ng} / \mathrm{mL}$ vs group $2=131.3(36.7-368.5)$ $\mathrm{ng} / \mathrm{mL}, \mathrm{p}=0.004$ ) as compared to group 1 .

In the female population, there was no comparison between the two groups since the small number of patients in group 2 (4 patients) was irrelevant in terms of statistical analysis.

Using a multiple regression model we found that ESA requirement is associated with higher BVP, DPB, nPCR, BMI and lower SBP and $\operatorname{UDV}\left(\mathrm{R}^{2}=0.2369\right.$, Adjusted $\mathrm{R}^{2}=0.1973$, $\mathrm{F}(18,347)=5.9842, \mathrm{p}<0.001$, Std.Error of estimate: 0.4329) (Table 2).

\section{Discussion}

A higher $\mathrm{Hb}$ target (such as $13.5 \mathrm{~g} / \mathrm{dL}$ ) achieved by using ESA in HD patients was not found to be associated with an improvement in quality of life or in a reduced number of adverse events such as myocardial infarction, congestive heart failure, stroke, or mortality 
Table 1. Clinical and laboratory parameter comparisons between the study groups

\begin{tabular}{lccc}
\hline Parameter & Group 1 (ESA) & Group 2 (non-ESA) & p \\
\hline Albumin (g/dL) & $3.7 \pm 0.4$ & $3.8 \pm 0.2$ & $\mathbf{0 . 0 3}$ \\
\hline BMI (kg/m $)$ & $27.6 \pm 5.4$ & $26.9 \pm 3.5$ & 0.17 \\
\hline Calcium (mg/dL) & $8.3 \pm 0.9$ & $9 \pm 0.8$ & $<\mathbf{0 . 0 0 1}$ \\
\hline Ferritin (ng/mL) & $486(303.4-844.6)$ & $131.3(26.7-393.8)$ & $<\mathbf{0 . 0 0 1}$ \\
\hline Phosphate (mg/dL) & $5.4 \pm 1.5$ & $5.6 \pm 1.2$ & 0.29 \\
\hline Hb (g/dL) & $11.1 \pm 1.32$ & $13 \pm 1.4$ & $<\mathbf{0 . 0 0 1}$ \\
\hline IBWG (\%) & $2.1 \pm 1.1$ & $2.4 \pm 1.2$ & $\mathbf{0 . 0 0 8}$ \\
\hline PTH (pg/mL) & $391.7(176-641.3)$ & $417(243.4-847.2)$ & 0.30 \\
\hline Kt/V & $1.5 \pm 0.3$ & $1.4 \pm 0.2$ & 0.05 \\
\hline TSAT (\%) & $31.2 \pm 13.9$ & $24.3 \pm 14.8$ & $\mathbf{0 . 0 0 9}$ \\
\hline SBP (mmHg) & $136.2 \pm 19.2$ & $131.5 \pm 19.4$ & $\mathbf{0 . 0 3}$ \\
\hline DBP (mmHg) & $73 \pm 12.4$ & $73.8 \pm 12.7$ & 0.45 \\
\hline UDV & $33.1 \pm 3.85$ & $34.8 \pm 2.5$ & $<\mathbf{0 . 0 0 1}$ \\
\hline BVP $(\mathbf{L})$ & $78.2 \pm 16.6$ & $81.9 \pm 13.6$ & $\mathbf{0 . 0 3}$ \\
\hline Weekly treatment time (min) & $720(720-780)$ & $720(720-720)$ & 0.80 \\
\hline nPCR & $1.1 \pm 0.3$ & $1.1 \pm 0.2$ & 0.98 \\
\hline CRP (mg/L) & $11.03(5.9-12.8)$ & $7.3(2.5-8.8)$ & $\mathbf{0 . 0 2 2}$ \\
\hline
\end{tabular}

ESA- erythropoiesis-stimulating agents, BMI- body mass index, Hb- hemoglobin, IBWG- interdialytic body weight gain, PTHintact parathormone, Kt/V - spKt/V -K/DOQI formula, TSAT- transferrin saturation, SBP- predialysis systolic blood pressure, DBP- predialysis diastolic blood pressure, UDV- urea distribution volume, BVP- blood volume processed, nPCR - normalized protein catabolic ratio, CRP - C-reactive protein

$(6,7)$. Patients with higher chance to maintain $\mathrm{Hb}$ levels above $12 \mathrm{~g} / \mathrm{dL}$ without ESA were men, of a lower age and with a higher dialysis vintage $(8,9)$. The use of a percutaneous HD catheter was associated with a lower likelihood of being part of the non-ESA group. The presence of ADPKD correlated with higher levels of $\mathrm{Hb}$ than other underlying kidney diseases and a higher chance of patients belonging to the non-ESA group $(2,10)$. In another study on 1318 HD patients (1307 in the ESA group and 11 in the non-ESA group), the non-ESA group had, again, a higher proportion of men, with a higher dialysis vintage, a higher prevalence of ADPKD, but also an increased level of endogenous EPO. There was no significant differ- ence in iron metabolism between the ESA and non-ESA group (11). Verdalles et al. compared 45 patients on HD belonging to the non-ESA group and 205 patients on HD belonging to the ESA group. Those who had non-ESA status were mostly younger men, with longer dialysis vintage, and a higher prevalence of ADPKD. When comparing ADPKD patients against subjects with acquired polycystic kidney disease, there was no significant difference in $\mathrm{Hb}$, dialysis vintage, age, gender, and EPO levels (3). Takeda et al. compared an ESA group that consisted of 133 patients against a non-ESA group that had 25 patients, and found no difference regarding gender, presence of ADPKD, serum albumin (increased in the non-ESA group that we 
Table 2. Association of clinical and laboratory parameters with ESA needs

\begin{tabular}{lcccc}
\hline Parameter & $\mathrm{b}^{*}$ & Std.Err. of $\mathrm{b}^{*}$ & $\mathrm{t}(347)$ & $\mathrm{p}$ \\
\hline Albumin $(\mathrm{g} / \mathrm{dL})$ & 0.061 & 0.053 & 1.162 & 0.246 \\
\hline BMI $\left(\mathrm{kg} / \mathrm{m}^{2}\right)$ & 0.320 & 0.072 & 4.445 & $<\mathbf{0 . 0 0 1}$ \\
\hline Calcium $(\mathrm{mg} / \mathrm{dL})$ & -0.081 & 0.058 & -1.414 & 0.158 \\
\hline Ferritin $(\mathrm{ng} / \mathrm{mL})$ & 0.028 & 0.053 & 0.520 & 0.604 \\
\hline Phosphate $(\mathrm{mg} / \mathrm{dL})$ & -0.089 & 0.056 & -1.593 & 0.112 \\
\hline Hb $(\mathrm{g} / \mathrm{dL})$ & 0.017 & 0.059 & 0.290 & 0.772 \\
\hline IBWG $(\%)$ & -0.089 & 0.052 & -1.716 & 0.087 \\
\hline PTH $(\mathrm{pg} / \mathrm{mL})$ & -0.063 & 0.049 & -1.279 & 0.202 \\
\hline Kt/V & -0.079 & 0.063 & -1.261 & 0.208 \\
\hline TSAT $(\%)$ & -0.055 & 0.048 & -1.135 & 0.257 \\
\hline SBP $(\mathrm{mmHg})$ & -0.264 & 0.070 & -3.761 & $<\mathbf{0 . 0 0 1}$ \\
\hline DBP $(\mathrm{mmHg})$ & 0.295 & 0.075 & 3.918 & $<\mathbf{0 . 0 0 1}$ \\
\hline UDV & -0.373 & 0.082 & -4.574 & $<\mathbf{0 . 0 0 1}$ \\
\hline BVP $(\mathrm{L})$ & 0.243 & 0.059 & 4.141 & $<\mathbf{0 . 0 0 1}$ \\
\hline Weekly treatment time $(\mathrm{min})$ & -0.013 & 0.050 & -0.251 & 0.802 \\
\hline nPCR & 0.235 & 0.060 & 3.918 & $<\mathbf{0 . 0 0 1}$ \\
\hline CRP $(\mathrm{mg} / \mathrm{L})$ & -0.034 & 0.049 & -0.710 & 0.478 \\
\hline
\end{tabular}

ESA- erythropoiesis-stimulating agents, BMI- body mass index, Hb- hemoglobin, IBWG- interdialytic body weight gain, PTH- intact parathormone, Kt/V - spKt/V -K/DOQI formula, TSAT- transferrin saturation, SBP- predialysis systolic blood pressure, DBP- predialysis diastolic blood pressure, UDV- urea distribution volume, BVP- blood volume processed, nPCR normalized protein catabolic ratio, CRP - C-reactive protein

studied), with another study finding again that non-ESA patients had higher albumin levels (2), iron values, C-reactive protein, nPCR (no difference as well in our study), and even endogenous EPO. However, higher dialysis vintage, higher BMI, and serum leptin levels were found to be independent factors that contribute to the presence of non-ESA status (we did not find differences in BMI) (12). When comparing the two groups in our study, there was no significant difference in age and dialysis vintage, but there was one regarding gender distribution, with more men belonging to the non-ESA group. This gender difference in erythropoiesis is caused by, among others, the stimulatory effect of androgens in the bone marrow, stimulation of EPO production by androgens in the kidneys, while estrogen has an inhibitory effect on red cell production in the bone marrow (13).

According to Shah et al., in patients with ADPKD with infrequent ESA usage (less than $25 \%$ of the follow-up time), a $\mathrm{Hb}$ value above 13 $\mathrm{g} / \mathrm{dL}$ offers greater survival, and in patients with ADPKD and regular ESA usage (greater than $25 \%$ of the follow-up time) an $\mathrm{Hb}$ between 12 and $13 \mathrm{~g} / \mathrm{dL}$ has the greater survival value. Also, in the lower $\mathrm{Hb}$ range, ADPKD patients have better survival than non-ADPKD ones (14).

Hypertension is an early sign in ADPKD that precedes the onset of actual renal failure and is usually diagnosed in the third decade of life, yet it is also common in children and young adults with ADPKD (15). We measured the SBP and DBP and found that only the SBP was signifi- 
cantly lower in the non-ESA group. Goodkin et al. found that hypertension in non-ESA patients was less common than in patients who need ESA (1) yet this result can be explained by the fact that ESA are in themselves a cause for hypertension, an effect that can be independent of the actual increase in hematocrit. In individuals on HD who receive ESA, SBP increased with an average of 5 to $8 \mathrm{mmHg}$ and DBP increased with 4 to $6 \mathrm{mmHg}$, as did the hospital admission rate for severe hypertension. The effect on blood pressure was dose-dependent (16).

Chronic inflammation in ESRD is considered one of the main factors that leads to increased ferritin values in patients on HD (17). ESA administration was associated with higher CRP compared to patients with no ESA treatment, confirming a role of inflammation in anemia pathogenesis. Increased ferritin in ESRD was also associated with a partial, inefficient response to ESA, and more severe anemia (18). Our non-ESA patients had lower values of ferritin than the ones in the ESA group, a result that matches others $(2,12)$. TSAT was also reduced in our group, as was in another study, while others did not observe a significant difference or did not find TSAT values as predictive factors for nonESA status $(2,3,12)$. The study by Gaweda et al. analyzed how ferritin and TSAT values influence $\mathrm{Hb}$ increase after ESA therapy in patients on HD. It was found that in lower TSAT ranges, $\mathrm{Hb}$ increase is greater the higher TSAT is. This effect reached a plateau in the $30-40 \%$ value range of TSAT and any further increase of it did not bring an added benefit. For ferritin, the maximum $\mathrm{Hb}$ response appeared at values of $1300 \mathrm{ng} / \mathrm{mL}$, yet $92 \%$ of the maximum $\mathrm{Hb}$ increase appeared at a ferritin value of $500 \mathrm{ng} / \mathrm{mL}$ and TSAT of $34 \%$. Even so, of the two markers, only TSAT was found to be a statistically significant predictor of $\mathrm{Hb}$ response to ESA, but not ferritin (19).

It has been observed that secondary hyperparathyroidism is involved in the pathogenesis of anemia in patients with ESRD. High PTH may induce bone marrow fibrosis, directly inhibit erythropoiesis, or increase the fragility of red blood cells. Pharmacological and surgical treatment of secondary hyperparathyroidism has been shown to improve anemia (20). In the two groups we studied, PTH and phosphate serum values were not significantly different, but serum calcium was higher in our non-ESA group. Others found that PTH was actually higher in the non-ESA group although it was not an independent factor for non-ESA status (12).

In a previous work IBWG did not present a significant correlation with $\mathrm{Hb}$ levels in patients on $\mathrm{HD}$, but $\mathrm{Hb}$ levels higher than $12 \mathrm{~g} / \mathrm{dL}$ were associated with cardiovascular mortality in subjects with an IBWG higher than 5.4\% (21). In our study non-ESA patients presented with higher IBWG values. IBWG was increased in men more than it was in women (22), and since our non-ESA group had a higher ratio of men it might explain the difference.

Regarding dialysis adequacy and ESA status we did not find differences between the two ADPKD groups. Other studies did not find a significant difference in $\mathrm{Kt} / \mathrm{V}$ and it was not a predictive factor for non-ESA status $(2,3)$. In a group of $68 \mathrm{HD}$ patients, $\mathrm{Kt} / \mathrm{V}$ did not correlate with hematocrit but a significant inverse association between Kt/V and ESA dose was observed. The ESA dose was significantly lower in patients with a Kt/V level above 1.4 when compared to patients with $\mathrm{Kt} / \mathrm{V}$ below 1.4 , with a $45 \%$ reduction in the ESA dose required (23).

The significant associations of ESA requirement revealed by multiple regression showed increased BVP, nPCR and BMI as predictive factors for ESA use. This is rather surprising since increased hemodialysis efficiency and better nutrition are generally known as correcting factors of renal anemia. ESA treatment is sometimes accompanied by hypertension. In our study higher DBP and lower SBP were associated with ESA 
requirement, suggesting a different tendency in patients with ADPKD. Better nourished ADPKD patients on HD, with a slightly better SBP control, with longer dialysis time and increased $\mathrm{Hb}$ had a lower ESA requirement in our study.

The limitation of this study consists of the relatively small number of patients, who, however, represent a significant group for ADPKD. Since the power of the multiple regression model was weak, the findings should be carefully interpreted.

\section{Conflict of interest}

The authors declare that they have no conflict of interest.

\section{References}

1. Pop ND, Bacârea A, Coroș L, Aloiziu Dogaru G, Hosu I, Bacârea V, et al. Serum erythropoietin level in anemia of chronic kidney disease - experience of a Romanian medical center. Rev Romana Med Lab 2015;23(1):97106. DOI: $10.1515 / \mathrm{rrlm}-2015-0003$

2. Goodkin DA, Fuller DS, Robinson BM, Combe C, Fluck R, Mendelssohn D, et al. Naturally occurring higher hemoglobin concentration does not increase mortality among hemodialysis patients. J Am Soc Nephrol 2011 Feb;22(2):358-65. DOI: 10.1681/ASN.2010020173

3. Verdalles U, Abad S, Vega A, Ruiz Caro C, Ampuero J, Jofre R, et al. Factors related to the absence of anemia in hemodialysis patients. Blood Purif 2011;32(1):6974. DOI: $10.1159 / 000323095$

4. Pisoni RL, Bragg-Gresham JL, Young EW, Akizawa $\mathrm{T}$, Asano Y, Locatelli F, et al. Anemia management and outcomes from 12 countries in the Dialysis Outcomes and Practice Patterns Study (DOPPS). Am J Kidney Dis 2004 Jul;44(1):94-111. DOI: 10.1053/j. ajkd.2004.03.023

5. Eckardt KU, Mollmann M, Neumann R, Brunkhorst R, Burger HU, Lonnemann G, et al. Erythropoietin in polycystic kidneys. J Clin Invest 1989 Oct;84(4):11606. DOI: $10.1172 / J C I 114280$

6. Singh AK, Szczech L, Tang KL, Barnhart H, Sapp S, Wolfson M, et al. Correction of anemia with epoetin alfa in chronic kidney disease. N Engl J Med 2006
Nov;355:2085-98. DOI: 10.1056/NEJMoa065485

7. Drüeke TB, Locatelli F, Clyne N, Eckardt KU, Macdougall IC, Tsakiris D, et al. Normalization of hemoglobin level in patients with chronic kidney disease and anemia. N Engl J Med 2006 Nov;355(20):2071-84. DOI: 10.1056/NEJMoa062276

8. Orasan OH, Orasan RA, Patiu MI, Dumitrascu DL. Hyaluronic acid in end-stage renal disease treated by hemodialysis. Renal failure 2015;37(9):1531-2. DOI: 10.3109/0886022X.2015.1077312

9. Orasan OH, Sava M, Iancu M, Cozma A, Saplontai-Pop A, Sarlea Tarmure S, et al. Serum hyaluronic acid in chronic viral hepatitis $\mathrm{B}$ and $\mathrm{C}$ : a biomarker for assessing liver fibrosis in chronic hemodialysis patients. International Urology and Nephrology 2015 Jul;47(7), 1209-17. DOI: 10.1007/s11255-015-1017-x

10. Locatelli F, Altieri P, Andrulli S, Sau G, Bolasco P, Pedrini LA, et al. Predictors of haemoglobin levels and resistance to erythropoiesis-stimulating agents in patients treated with low-flux haemodialysis, haemofiltration and haemodiafiltration: results of a multicentre randomized and controlled trial. Nephrol Dial Transplant 2012 Sep;27(9):3594-600. DOI: 10.1093/ndt/gfs117

11. Wan Q, He Y, Zhang W, Wu Q, Xiong Z. Prevalence and related factors of the absence of anemia among Chinese chronic hemodialysis patients: a multicenter cross-sectional study. Int Urol Nephrol 2014 Aug;46(8):16514. DOI: $10.1007 / \mathrm{s} 11255-013-0581-1$

12. Takeda A, Toda T, Shinohara S, Mogi Y, Matsui N. Factors contributing to higher hematocrit levels in hemodialysis patients not receiving recombinant human erythropoietin. Am J Kidney Dis 2002 Jul;40(1):104-9. DOI: 10.1053/ajkd.2002.33918

13. Murphy WG. The sex difference in haemoglobin levels in adults - mechanisms, causes, and consequences. Blood Rev 2014 Mar;28(2):41-7. DOI: 10.1016/j. blre.2013.12.003

14. Shah A, Molnar MZ, Lukowsky LR, Zaritsky JJ, Kovesdy CP, Kalantar-Zadeh K. Hemoglobin level and survival in hemodialysis patients with polycystic kidney disease and the role of administered erythropoietin. Am J Hematol 2012 Aug;87(8):833-6. DOI: 10.1002/ ajh. 23255

15. Rahbari-Oskoui F, Williams O, Chapman A. Mechanisms and management of hypertension in autosomal dominant polycystic kidney disease. Nephrol Dial Transplant 2014 Dec;29(12):2194-201. DOI: 10.1093/ 
$\mathrm{ndt} / \mathrm{gft} 513$

16. Krapf R, Hulter HN. Arterial hypertension induced by erythropoietin and erythropoiesis-stimulating agents (ESA). Clin J Am Soc Nephrol 2009 Feb;4(2):47080. DOI: $10.2215 /$ CJN.05040908

17. Orasan RA, Patiu IM, Anghel D, Bejan C, Iosub L, Totolici C, et al. Variation of clinical and laboratory features in chronic dialysis patients treated with high-flux hemodialysis after switching to online hemodiafiltration. Int Urol Nephrol 2013 Oct;45(5):1415-22. DOI: 10.1007/s11255-012-0341-7

18. Kalantar-Zadeh K, Kalantar-Zadeh K, Lee GH. The fascinating but deceptive ferritin: to measure it or not to measure it in chronic kidney disease? Clin J Am Soc Nephrol 2006 Sep;1(Suppl 1):9-18.6

19. Gaweda AE, Bhat P, Maglinte GA, Chang CL, Hill J, Park GS, et al. TSAT is a better predictor than ferritin of hemoglobin response to Epoetin alfa in US dialysis patients. Hemodial Int 2014 Jan;18(1):38-46. DOI: 10.1111/hdi.12078
20. Komaba H, Kakuta T, Fukagawa M. Management of secondary hyperparathyroidism: how and why? Clin Exp Nephrol 2017 Mar;21(Suppl 1):37-45. DOI: 10.1007/s10157-016-1369-2

21. Toida T, Iwakiri T, Sato Y, Komatsu H, Kitamura K, Fujimoto S. Relationship between Hemoglobin Levels Corrected by Interdialytic Weight Gain and Mortality in Japanese Hemodialysis Patients: Miyazaki Dialysis Cohort Study. PLoS One 2017 Jan;12(1):e0169117. DOI: 10.1371/journal.pone.0169117

22. Artan AS, Kircelli F, Ok E, Yilmaz M, Asci G, Dogan C, et al. Dialyzing women and men: does it matter? An observational study. Clin Kidney J 2016 Jun;9(3):48693. DOI: $10.1093 / \mathrm{ckj} / \mathrm{sfw} 013$

23. Movilli E, Cancarini GC, Zani R, Camerini C, Sandrini M, Maiorca R. Adequacy of dialysis reduces the doses of recombinant erythropoietin independently from the use of biocompatible membranes in haemodialysis patients. Nephrol Dial Transplant 2001 Jan;16(1):111-4. DOI: $10.1093 / \mathrm{ndt} / 16.1 .111$ 